\title{
Influences on Achieving Motor Milestones: A Twin-Singleton Study
}

\author{
Silvia I. Brouwer,' Toos C. E. M. van Beijsterveldt,' Meike Bartels,' James J. Hudziak, ${ }^{2}$ and Dorret I. Boomsma' \\ ' Department of Biological Psychology,Vrije Universiteit, Amsterdam, the Netherlands \\ ${ }^{2}$ Departments of Psychiatry and Medicine (Division of Human Genetics), Center for Children, Youth and Families, University of Vermont, \\ College of Medicine Burlington, Burlington, Vermont, United States of America
}

\begin{abstract}
In order to determine if twinning impacted achievement of motor milestones the attainment of early motor milestones in twins was examined and compared to published data from singletons of the same age from the same culture and birth years. We examined the influence of twinning, sex, zygosity and birth cohort (1987-2001) on the motor development of twins aged 0 to 24 months. Data on the attainment of motor milestones (turn, sit, crawl, stand and walk) of twins were collected from maternal reports. All data were corrected for gestational age. Data from the twin sample were compared to normative data from singletons, which were available from Child Health Clinics ( $\mathrm{CHC}$ ). Analyses across twin data and the $\mathrm{CHC}$ singleton data revealed no differences between twins and singletons in achievement of motor milestones. Girls were able to sit without support slightly earlier than boys, otherwise there were no other sex differences. Birth-order analyses revealed minimal but significant differences in turning over from back to belly and for sitting without support between the first- and second-born. Dizygotic (DZ) twins were faster than monozygotic (MZ) twins in achieving the moment of sit, crawl, stand and walk. Twins born in earlier cohorts were faster in reaching the moment of crawl, sit and walk. It is concluded that there are no differences in time of reaching motor milestones between twins and singletons within the normal range. Sex has minimal to no effect on motor development in early childhood. DZ twins achieve motor milestones sooner than $\mathrm{MZ}$ twins. Attainment of gross motor milestones (crawl, stand and walk) is delayed in later birth cohorts.
\end{abstract}

Twin studies have been fundamental to the investigation of genetic contributions to multiple phenotypes, whether they be quantitative (e.g., IQ, height) or categorical (e.g., attention-deficit/hyperactivity disorder). In the field of behavioral genetics, twin populations have been used to study the genetic and environmental contributions to a variety of, for example, cognitive, personality and psychiatric traits or illnesses. The data that result from these studies are used to drive family and sib-pair analyses and hunt for specific genetic and environmental factors. There is, however, debate about the utility of the twin model in the study of human behavior. Are the results generalizeable to nontwin populations? With recent reports of gene-environment interactions, and epigenetic phenomena in the etiology of psychopathology, it is reasonable to ask just how closely data from twin studies relate to singleton data. One proxy for testing the environmental effects of twin births is to relate early developmental milestones between twins and singletons to determine twin behavior deviates from that of singletons. One such method would be to test the achievement of major developmental milestones of twins and singletons and determine the relative variance across these groups and then within twins, by birth order, sex, and zygosity status.

The rationale for this work is relatively straightforward. Twins weigh less than singletons even when born full term (born between 36 and 41 weeks of gestation; Liu \& Blair, 2002; Luke et al., 1991; Min et al., 2000). Twins are born preterm more often than singletons. Several studies report that a delay in motor development is associated with low birthweight and shorter gestational age (GA; Allen \& Alexander, 1990; Cheung et al., 2001; Den Ouden et al., 1991; Goyen \& Lui, 2002; Lems et al., 1993; Lui et al., 2001; Piper et al., 1989). Therefore, when studying the early motor development in twins, GA should be taken into account (Peter et al., 1999).

The attainment of gross motor milestones is an important indicator of child development. Such milestones include turning over from back to belly (turn), crawling on hands and knees (crawl) and sitting (sit), standing (stand) and walking (walk) without support (Levitt, 1986; Livshits \& Peter, 1996; Thelen, 2000). Attainment of these milestones may be of clinical importance for detecting delays. Delays in motor milestone achievement are thought to be an indicator

\section{Received 14 October, 2005; accepted 17 February, 2006.}

Address for correspondence: S. I. Brouwer, Vrije Universiteit, Department of Biological Psychology, Van der Boechorststraat 1, 1081 BT, Amsterdam, the Netherlands.E-mail: si.brouwer@psy.vu.nl 
of abnormal development. It is therefore important to identify the factors that influence the normal range of attainment for motor milestones. In Child Health Clinics (CHC) in the Netherlands, reference values of motor milestones of 'het van Wiechenschema' are used (turn $=6$ months; sit $=9$ months; crawl $=12$ months; stand $=15$ months; walk $=18$ months) to screen infants for possible delays. These values were derived from a study carried out in the early 1970 s by Schlesinger-Was (1981). Schlesinger-Was reported the age upon which $90 \%$ of the infants reached a certain milestone. To establish whether these reference values were still useful in the 1990s, Verkerk and colleagues (1993) used data on motor milestones from the 'Social Medical Survey of Children Attending Child Health Clinics' (SMOCC) study (Herngreen et al., 1992) to investigate reference values used by Schlesinger-Was. The SMOCC is a population-based, observational, follow-up study of a cohort of Dutch children investigated from birth to 24 months of age. Good agreement was found between the reference values in the SMOCC study and the values reported at the $\mathrm{CHC}$. As these data are primarily from singletons it is important to determine if these same reference values are useful for twins. This investigation is the first goal of this report. We will determine if reference values developed primarily in the study of singleton births are applicable to twin births.

Several studies have shown sex differences in motor development during childhood and adolescence as reviewed by Thomas and French (1985). But when these changes in motor development emerge is still uncertain. Within our sample of infant twins aged between 0 and 24 months we will examine if sex differences exist during the first 2 years of life. As monozygotic (MZ) twins are seen as more vulnerable than dizygotic (DZ) twins due to an excess of structural defects (Cao \& Monni, 2005; Luke \& Keith, 1990; Schnitzel et al., 1979) an effect of zygosity on motor development is also examined in this report.

Data of gross motor milestones have been collected in the last 15 years (1986-2001) by the Netherlands Twin Register (NTR). We divided all twins into different birth cohorts to examine possible cohort effects. Due to a change in clinical practice which changed the advise for sleep position in the early 1990s, a change might be expected in the time of attaining motor milestones since parents are advised to put their children to sleep in the supine (on the back) position rather than the prone (on the belly) position. Several studies have stated the effect of sleep position on motor development (Davis et al., 1998; Dewey et al., 1998; Jantz et al., 1997; Ratliff-Schaub et al., 2001; Salls et al., 2002; Visscher et al., 1998). Prone sleepers/players attain several motor milestones earlier than supine sleepers/players.

To summarize, we examined the early motor development of healthy twin pairs from the NTR born between 1987 and 2001. To see whether motor development of twins is comparable to that of singletons in the Netherlands, we compared our data of motor milestones from twins with the SMOCC study. We looked at the effects of sex, birth order, zygosity and birth cohort on measures of motor development of twins corrected for GA.

\section{Methods \\ Subjects}

This study is part of a longitudinal study on early child development. Data on 11,712 pairs of twins born between 1986 and 2001 were obtained from surveys collected by the NTR, with questions about zygosity, birthweight, GA and motor milestones. Forty to fifty per cent of all multiple births in the Netherlands are registered by the NTR (Boomsma, 1998; Boomsma et al., 1992). Participation in research is voluntarily. For this study on motor development two hundred 48 pairs were excluded because either one or both of the children had a disease or serious mental or physical disability which could influence the development. Since this article concerns motor development, we also left out 73 twin pairs with milder physical disabilities like clubfoot, hip deviation and hypo- or hypertension in muscles. A relative healthy group of 11,391 pairs of twins remained for analysis. From these twin pairs $3773(33.1 \%)$ were MZ and $7618(66.9 \%)$ were DZ. Information about the zygosity of twins was obtained from questions in questionnaires collected at the age of 3 and/or 5, 7, 10, 12 years (Rietveld et al., 2000) and from Blood/DNA typing $(N=1059)$. There were 11,357 $(49.9 \%)$ boys and $11,417(50.1 \%)$ girls. The 11,391 pairs of twins born between 1987 and 2001 were subdivided into five cohorts $(1987-1989=$ cohort 1 , 1990-1992 = cohort $2,1993-1995=$ cohort 3 , 1996-1998 = cohort 4 and 1999-2001 = cohort 5).

To test if motor development of twins is comparable to the motor development of singletons we selected a group of twins from this study comparable to the cohort of children used in the SMOCC study. The SMOCC study is a population-based, observational, follow-up study of a cohort of children born 1988 to 1989. The SMOCC birth cohort consisted of 2151 live-born infants ( $49.2 \%$ boys, $50.8 \%$ girls). To establish reference values for healthy children some groups were excluded: children from non-West European countries; children with a developmental disorder detected at $\mathrm{CHC}$ and confirmed by specialists; children with a disorder which can influence their development from average (like clubfoot or spasm); and children with birthweight less than 2500 grams or GA less than 37 weeks. Children in the SMOCC study were examined by the physician and the district nurse at the ages of $1,2,3,6,9,12,15,18$ and 24 months in CHC. The physician or district nurse reported if a motor milestone was obtained during these examinations. A twin sample matched on year of birth (1988-1991), birthweight (> 2500 grams) and GA (> 36.5 weeks) was selected from the NTR. 
Table 1

Percentage Twins and Singletons Who Reached Motor Milestones by a Fixed Age

\begin{tabular}{|c|c|c|c|c|c|c|}
\hline Motor milestone & $\begin{array}{l}\text { MZ twins } \\
\text { NTR }(n)\end{array}$ & $\begin{array}{c}\text { Percentage } \\
\text { MZ twins }\end{array}$ & $\begin{array}{l}\text { DZ twins } \\
\text { NTR ( } n\end{array}$ & $\begin{array}{c}\text { Percentage } \\
\text { MZ twins }\end{array}$ & $\begin{array}{l}\text { Singletons } \\
\text { SMOCC }(n)\end{array}$ & $\begin{array}{c}\text { Percentage } \\
\text { singletons }\end{array}$ \\
\hline \multicolumn{7}{|l|}{ Turn } \\
\hline 9 months & $719 / 747$ & $96 \%$ & $1499 / 1554$ & $97 \%$ & $970 / 1039$ & $93 \%$ \\
\hline \multicolumn{7}{|l|}{ Sit } \\
\hline 12 months & $761 / 770$ & $99 \%$ & $1578 / 1594$ & $99 \%$ & $1046 / 1059$ & $99 \%$ \\
\hline \multicolumn{7}{|l|}{ Crawl } \\
\hline 15 months & $735 / 749$ & $98 \%$ & $1556 / 1581$ & $98 \%$ & $871 / 912$ & $96 \%$ \\
\hline \multicolumn{7}{|l|}{ Walk } \\
\hline 18 months & $752 / 786$ & $96 \%$ & $1576 / 1645$ & $96 \%$ & $822 / 860$ & $96 \%$ \\
\hline
\end{tabular}

\section{Measures}

Information from the twins on birthweight and GA was obtained by maternal rating from a first questionnaire which was sent shortly after the twins were born. Birthweight was measured by a doctor or nurse shortly after birth. Parents were asked to report these birthweights. With this questionnaire parents received a list to keep track of important motor milestones (turn, sit, crawl, stand, walk). In a second questionnaire, mailed out when the twins were at the age of 2, parents were asked to report the age in which the motor milestones were reached. Those motor milestones were turning over from back to belly (turn), sitting without support (sit), crawling on hands and knees (crawl), standing without support (stand) and walking without support (walk). Twin data on motor milestones were compared with data on motor milestones of singletons according to reference values of 'het van Wiechenschema'. Missing values for each motor milestone were between $2.3 \%$ and $9.7 \%$.

\section{Statistical Analyses}

Differences between first- and second-born were tested by paired $t$ tests. As data from twins are not independent, we only present analyses based on data of the first-born twin (we only noted a small difference for obtaining the moment of turn and sit) on motor milestones. The Statistical Package for Social Science 11.5 (SPSS) was used to analyze the data. Frequency analyses were used to compare the percentage of twins and singletons that attained TURN within 9 months, SIT within 12 months, CRAWL within 15 months and WALK within 18 months. Cross-tabulations were used for analysis of the percentages $M Z$ and $\mathrm{DZ}$ twins in different cohorts.

For every motor milestone, means of the motor milestone from $\mathrm{MZ}$ and $\mathrm{DZ}$ twins were compared over different cohorts. ANOVA was carried out with sex (male/female), zygosity (MZ/DZ) and cohort (1-5) as between factors and GA as a covariate. Because of the high correlation between GA and birthweight we only used GA as a covariance.
To test whether girls from same-sex pairs (FF) differed from girls of opposite-sex pairs (FM/MF), and likewise if boys from same-sex pairs (MM) differed from boys of opposite-sex pairs, ANOVA was carried out both for the group of girls and the group of boys with same-sex or opposite-sex pair as a factor.

\section{Results \\ Twins Versus Singletons}

Table 1 shows the percentage of singletons and a selected group of MZ and DZ twins who reached a certain motor milestone (turn, sit, crawl and walk) at a fixed moment $(9,12,15$ and 18 months, respectively) as used by 'het van Wiechenschema'. For turn, sit, crawl and walk a minimum frequency of $90 \%$ of the twins had reached the motor milestones at the age of 9 , 12, 15 and 18 months, respectively. Comparison between $\mathrm{MZ}$ and $\mathrm{DZ}$ twins and singletons who reached a certain motor milestone at a fixed moment showed a few small differences. For turn and crawl the proportion $\mathrm{MZ}$ and $\mathrm{DZ}$ twins who reached these milestones at a fixed moment were slightly higher than singletons.

There was a significant decrease in GA over the cohorts $(p<.001)$. This linear effect went from 37.1 weeks in cohort 1 to 36.5 weeks in cohort 5 . Conforming to other studies (GA; Allen \& Alexander, 1990; Cheung et al., 2001; Den Ouden et al., 1991; Goyen \& Lui, 2002; Lems et al., 1993; Lui et al., 2001; Piper et al., 1989) we found that the effect of GA was significant for all motor milestones $(p<.001)$. Pearson correlations were -.246 for turn, -.249 for sit, -.191 for crawl, -.236 for stand, and -.246 for walk. Therefore we used GA as a covariate in all analyses and results. There was a highly significant correlation between birthweight and GA of .736 $(p<.001)$. Because of this high correlation we only used GA as a covariate.

\section{Sex and Birth Order}

Girls were faster than boys $(p<.001)$ for sit only. There were no differences between DZ girls from same-sex or opposite-sex twin pairs (DOS). For DZ boys from same-sex or DOS pairs no differences were 
found either. There were some small, but significant differences between first- and second-born twins. First-born twins were faster than second-born twins for turn $(p=.001)$ and sit $(p=.003)$. These differences in means were only less than .043 months $(<1.5$ days $)$. For crawl, stand and walk no differences were found. Therefore we report for all other analyses on data of the first-born twin.

\section{Zygosity}

There was a significant increase in the percentage of DZ twin pairs over the cohorts $(p<.001)$ ranging from $62.8 \%$ in cohort 1 to $70.3 \%$ in cohort 5 . Differences for mean GA between MZ and DZ twin pairs were significant $(p<.001)$. MZ twin pairs are born at 36 weeks and 3 days, DZ twin pairs are born at 36 weeks and 6 days. The percentage of twins who were born before 32 weeks was $3.8 \%$ for DZ twins and $4.5 \%$ for $\mathrm{MZ}$ twins.

DZ twins were faster than $\mathrm{MZ}$ twins in reaching the moment for sit $(p<.001)$, crawl $(p=.013)$, stand $(p<.001)$ and walk $(p<.001)$, but not for turn.

\section{Cohort}

Between the five cohorts there were significant differences in the age of motor milestone achievement for turn $(p<.001)$, crawl $(p<.001)$, stand $(p<.001)$, and walk $(p<.001)$ as shown in Figure 1 . For sit there were no significant cohort effects. For crawl, stand and walk, twins from the latest cohorts were slower in motor milestone achievement than twins from the first cohorts. Differences between cohort 1 and cohort 5 were at average 0.4 months for crawl, 0.5 months for walk and 0.8 months for stand. For turn the effects of cohort went up and down. Differences were 0.3 months (= 9 days) at the top.

Figure 1 shows the results of motor milestone achievement in months for $\mathrm{MZ}$ male (MZM), female (MZF) and DZ male (DZM), female (DZF) for each motor milestone by cohort. All results presented are with adjustment for GA. In all cohorts the age of milestone achievement was significantly higher for MZ for sit $(p<.001)$, crawl $(p=.015)$, stand $(p<.001)$ and walk $(p=.001)$ but not for turn. Differences were up to 10 days.

\section{Discussion}

Our data suggest that there are no remarkable differences between healthy singletons and healthy twins in the achievement of gross motor milestones within the normal range in the Netherlands. Within the standards that are used in CHC (9 months for turn; 12 months for sit; 15 months for crawl; 18 months for walk) over $90 \%$ of the twins reached the moment of attainment for the gross motor milestones. The group of twins from the NTR who participated in this study are comparable to the group of singletons from the SMOCC study. However, the assessment of the data was different in both studies. For the motor milestone achievement in twins, parents or caregivers were asked to report the information by questionnaire when the twins were 2 years old. For the singletons in the SMOCC study, parents or caregivers were asked by a physician or district nurse if a specific motor milestone was achieved at the moment of visiting the $\mathrm{CHC}$.

The results of the comparison between the twins of the NTR and the singletons of the SMOCC study are in line with the study of Verkerk et al. (1993), which found that the frequency distribution of the SMOCC and reference values as used by Schlesinger-Was differences were smaller than $10 \%$. In a study of British twins born before 34 weeks GA (Morly et al., 1989), no differences were found between twins and singletons. However, a study of Goetghebuer et al. (2003) reports that motor development during the first 18 months of life was delayed in Gambian twins compared to singletons for eight motor milestones. However, after adjustment for birthweight and number of siblings, singletons were ahead for three out of eight motor milestones (maintain head, sit and walk). As only children with a birthweight over 2500 grams were enrolled in the Gambian study and pairs with small GA and low birthweight were excluded, twins had a significant higher GA than singletons. Moreover, the study used the age of attainment of gross motor milestones for twins and singletons, which is more accurate than the comparison in our study, which uses the percentage of twins who reached a certain motor milestone by a fixed age. This is a possible explanation for the different results. Using just the percentage of twins who achieved a certain motor milestone can be less accurate but catches the development within the standard range for normal motor development.

There was a significant effect of GA on time of achieving the motor milestones. Twins born before term were prone to reach their milestones later than twins who are born at term. The correction for GA performed was consistent with the literature where several studies found that gross motor development needs to be corrected in the first 12 to 18 months of life (Allen \& Alexander, 1990; Cheung et al., 2001; Den Ouden et al., 1991; Goyen \& Lui, 2002; Lems et al., 1993; Lui et al., 2001; Pedersen et al., 2000; Piper et al., 1989). Our study confirms earlier findings.

Except for reaching the moment for sitting without support, sex effects were not found. This is consistent with the findings of Livshits et al. (1992) in twins and Goyen and Lui (2002) and Hindmarsh et al. (2000) who did not find sex effects on early motor development in preterm infants. However, it does not exclude that sex differences in motor development may emerge at later ages.

$\mathrm{MZ}$ twins were slower than DZ twins in reaching the moment for sit, crawl, stand and walk but not for turn. A possible explanation for the differences between MZ and DZ twins that remain in motor development after correction for GA is the greater vulnerability of $\mathrm{MZ}$ twins. But given the standard ranges 

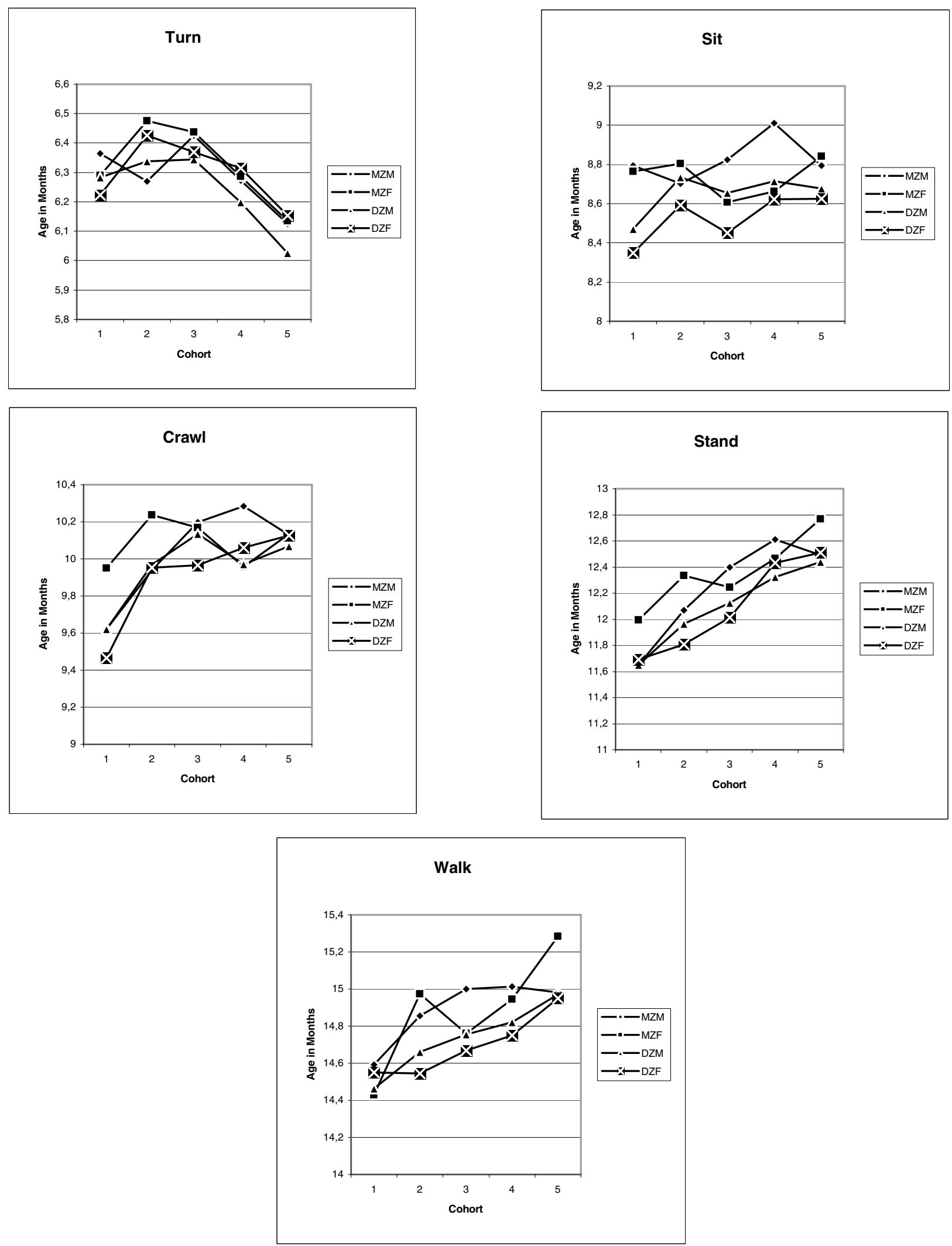

Figure 1

Motor milestone achievement in months for monozygotic male (MZM), female (MZF) and dizygotic male (DZM), female (DZF) by cohort.

Note: For each motor milestone (turn, sit, crawl, stand and walk) the means corrected for GA are reported in months for MZM, MZF, DZM and DZF (first-born twin). Dizygotic males/females are all males/females both from same-sex and opposite-sex twin pairs. For turn a decrease in months follows an increase from cohort 3 for all groups. For crawl, stand and walk an increase in months is reported by cohort for all groups. 
for motor milestone achievement as used in $\mathrm{CHC}$, Table 1 shows that MZ twins fall into the ranges of normal development. No differences were found for girls of the same sex compared to girls of opposite-sex twins. The same applies to boys. A second, behavioral possibility is that interaction is keener in DZ pairs. Observing your genetically different co-twin sit early may be motivating for infants, who use mimicry as a major form of communication. Although first-born twins were significantly faster than second-born twins in reaching the moment for turn and sit, but not for crawl, stand and walk, these differences were smaller than 1.5 days.

The effect of cohort on motor milestone achievement found in this study is remarkable. For crawl, stand and walk we found a significant effect of cohort, but the pattern was different with the pattern shown for turn. We noted an increase in time of achievement for crawl, stand and walk. Data shows that for crawl, stand and walk, twins in cohort 1 reached these milestones sooner than twins in cohort 5 , which means a delay in motor development. Differences between cohorts were up to 14 days for crawl, 16 days for walk and 25 days for stand. For sit we did not find any effect of cohort. For turn the effect of cohort was significant. A delay was first seen from cohort 1 up to cohort 3. But from cohort 3 up to cohort 5 twins achieved the moment of turn faster. These data argue for delaying the moment of delivery, if possible, in order to minimize the effects of GA on the attainment of milestones.

In the CHC in the Netherlands the time delay in crawl, stand and walk will probably fall within the normal ranges because they do not register the time (in months) of achievement of motor milestones in children but rather score at fixed moments if motor milestones are reached. One possible explanation for the delay in crawl, stand and walk may be the sleep position which is recommended by family doctors in the CHC. In the early 1990s, CHC recommended that healthy infants should be positioned on their back for sleep to decrease the incidence of sudden infant death syndrome (SIDS). Our data, which show a delay starting in the 1990s, and different studies which show an effect of sleep position on motor development (Davis et al., 1998; Dewey et al., 1998; Jantz et al., 1997; Ratliff-Schaub et al., 2001; Salls et al., 2002; Visscher et al., 1998;) suggest that prone sleeping may have positive effects on the motor development. Dewey et al. (1998) assume that the prone sleeper is stimulated to move and explore because the position itself is inherently boring and the child must do something to change these circumstances. Elaborating on this, Davis et al. (1998) suggest that supine sleepers lag behind prone sleepers in milestones that require the use of upper trunk. This upper-extremity muscle development occurs routinely in infants who spend more time in the prone position. No unambiguous explanations are given for this phenomenon. Another explanation is the arrival of the baby-walker, which also showed adverse effects on motor development (Siegel \& Burton, 1999) as baby walkers enable precocious locomotion in very young.

More detailed information and studies are necessary to find out if sleep position and other environmental changes cause structural effects of delay in the motor milestone achievement between cohorts. Therefore it needs to be considered if structural delays have importance in a child's future development.

\section{Acknowledgments}

Financial support by The Netherlands Organization for Scientific Research is gratefully acknowledged (NWO, grant number 575-25-012) \& (NWO/SPI 56464-14192). S. I. Brouwer is supported by National Institute of Mental Health (NIMH, RO1, MH5879903). M. Bartels is financially supported by NWO (VENI: 451-04-034).

\section{References}

Allen M. C., \& Alexander G. R. (1990). Gross motor milestones in preterm infants: Correction for degree of prematurity. Journal of Pediatrics, 116, 955-959.

Boomsma, D. I. (1998). Twin registers in Europe: An overview. Twin Research, 1, 34-51.

Boomsma, D. I., Orlebeke, J. F., \& Van Baal, G. C. M. (1992). The Dutch twin register: Growth data on weight and height. Behavior Genetics, 22, 247-251.

Cao, A., \& Monni, G. (2005). Phenotypic and genotypic discordance in monozygotic twins. In I. Blickstein \& L. G. Keith (Eds.), Multiple pregnancy (2nd edition; pp. 226-232). London: Taylor and Francis Group.

Cheung, Y. B., Yip, P. S. F., \& Karlberg, J. P. E. (2001). Fetal growth, early postnatal growth and motor development in Pakistani infants. International Journal of Epidemiology, 30, 66-74.

Davis, B. E., Moon, R. Y., Sachs, H. C., \& Ottolini, M. C. (1998). Effects of sleep position on infant motor development. Pediatrics, 102, 1135-1140.

Den Ouden, L., Rijken, M., Brand, R., VerlooveVanhorick, S. P., \& Ruys, J. H. (1991). Is it correct to correct? Developmental milestones in 555 'normal' preterm infants compared with term infants. Journal of Pediatrics, 118, 399-404.

Dewey, C., Fleming, P., Golding, J., \& The ALSPAC Study Team. (1998). Does the supine sleeping position have any adverse effects on the child? II. Development in the first 18 months. Pediatrics, 101, 1-5.

Goetghebuer, T., Ota, M. O., Kebbeh, B., John, M., Jackson-Sillah, D., Vekemans, J., Marchant, A., Newport, M., \& Weiss H. A. (2003). Delay in motor development of twins in Africa: A prospective cohort study. Twin Research, 6, 279-284.

Goyen, T. A., \& Lui, K. (2002). Longitudinal motor development of 'apparently normal' high-risk infants 
at 18 months, 3 and 5 years. Early Human Development, 70, 103-115.

Herngreen, W. P., Reerink, J. D., van Noord-Zaanstra, B. M., Verloove-Vanhorick, S. P., \& Ruys, J. H. (1992). SMOCC: Design of a representative cohort-study of live-born infants in the Netherlands. European Journal of Public Health, 2, 117-122.

Hindmarsh, G. J., O’Callaghan, M. J., Mohay, H. A., \& Rogers, Y. M. (2000). Gender differences in cognitive abilities at 2 years in ELBW infants. Early Human Development, 60, 115-122.

Jantz, J. W., Blosser, C. D., \& Fruechting, L. A. (1997). A motor milestone change noted with change in sleep position. Archives of Pediatrics and Adolescent Medicine, 151, 565-568.

Lems, W., Hopkins, B., \& Samson, J. F. (1993). Mental and motor development in preterm infants: The issue of corrected age. Early Human Development, 34, 113-123.

Levitt, S. (1986). Paediatric developmental therapy (chap. 2, pp. 13-33). Oxford: Blackwell.

Liu, X., Sun, Z., Neiderhiser, J. M., Uchiyama, M., \& Okawa, M. (2001). Low birth weight, developmental milestones, and behavioral problems in Chinese children and adolescents. Psychiatry Research, 101, 115-129.

Liu, Y. C., \& Blair, E. M. (2002). Predicted birthweight for singletons and twins. Twin Research, 5, 529-537.

Livshits, G., Cohen, Z., \& Otremski, I. (1992). Interrelations between early child development, gestational age and birth weight. International Journal of Anthropology, 7, 41-51.

Livshits, G., \& Peter, I. (1996). Family and biological factors in preterm birth and early child development in Israel. Journal of Ecology, 4, 115-124.

Luke, B., \& Keith, L. G. (1990). Monozygotic twinning as a congenital defect and congentital defects in monozygotic twins. Fetal Diagnosis and Therapy, 5, 61-69.

Luke, B., Witter, F. R., Abbey, H., Feng, T., Namnoum, A.B., Paige, D. M., \& Johnson, T. R. (1991). Gestational age-specific birthweights of twins versus singletons. Acta Geneticae Medicae et Gemellologiae, 40, 69-76.

Min, S. J., Luke, B., Gillespie, B., Min, L., Newman, R. B., Mauldin, J. G., Witter, F. R., Salman, F. A., \& O'Sullivan, M. J. (2000). Birth weight references for twins. American Journal of Obstetrics Gynecology, 182, 1250-1257.

Morly, R., Cole, T. J., Powell, R., \& Lucas, A. (1989). Growth and development in premature twins. Archives of Disease in Childhood, 64, 1042-1045.
Pedersen, S. J., Sommerfelt, K., \& Markestad, T. (2000). Early motor development of premature infants with birthweight less than 2000 grams. Acta Pediatrics, 89, 1456-1461.

Peter, I., Vainder, M., \& Livshits, G. (1999). Genetic analyses of motor milestones attainment in early childhood. Twin Research, 2, 1-9.

Piper, M. C., Byrne, P. J., Daraah, J., \& Watt, M. J. (1989). Gross and fine motor development of preterm infants at eight and 12 months of age. Developmental Medicine and Child Neurology, 31, 591-597.

Ratliff-Schaub, K., Hunt, C. E., Crowel, D., Golub, H., Smok-Pearsall, S., Palmer, P., Schafer, S., Bak, S., Cantey-Kiser, J., \& O’Bell, R. (2001). Relationship between infant sleep position and motor development in preterm infants. Journal of Developmental and Behavioral Pediatrics, 22, 293-299.

Rietveld, M. J. H., van der Valk, J. C., Bongers, I. L., Stroet, T. M., Slagboom, P. E., \& Boomsma, D. I. (2000). Zygosity diagnosis in young twins by parental report. Twin Research, 3, 134-141.

Salls, J. S., Silverman, L. N., \& Gatty, C. M. (2002). The relationship of infant sleep and play positioning to motor milestone achievement. American Journal of Occupational Therapy, 56, 577-580.

Schlesinger-Was, E. A. (1981). Ontwikkelingsonderzoek van zuigelingen en kleuters op het consultatiebureau [dissertatie]. Leiden: Rijksuniversiteit.

Schnitzel, A. A., Smith, D. W., \& Miller, J. R. (1979). Monozygotic twinning and structural defects. Journal of Pediatrics, 95, 921-930.

Siegel, A. C., \& Burton, R. V. (1999). Effects on baby walkers on motor and mental development in infants. Journal of Development and Behavioral Pediatric, 5, 355-361

Thelen, E. (2000). Motor development as foundation and future of developmental psychology. International Journal of Behavioral Development, 24, 385-397.

Thomas, J. R., \& French, K. E. (1985). Gender differences across age in motor performance A meta-analysis. Psychological Bulletin, 98, 260-282.

Verkerk, P. H., Reerink, J. P., \& Herngreen, W. P. (1993). Evaluatie van het van Wiechenschema. I De overeenkomst tussen referentiewaarden en waarnemingen in de praktijk. Tijdschrift voor Jeugdgezondheidszorg, 25, 71-77.

Visscher, F., van der Graaf, T., Spaans, M., van Lingen, R. A., \& Fetter, W. P. (1998). Prone position favors development of infants. Nederlands Tijdschrift voor Geneeskunde, 142, 2879-2880. 